\title{
Constructing Discrete Unbounded Distributions with Gaussian-Copula Dependence and Given Rank Correlation
}

\author{
Athanassios N. Avramidis \\ School of Mathematics, University of Southampton \\ Highfield, Southampton, SO17 1BJ, UNITED KINGDOM \\ aa1w07@soton.ac.uk
}

April 25, 2013

\begin{abstract}
A random vector $\mathbf{X}$ with given univariate marginals can be obtained by first applying the normal distribution function to each coordinate of a vector $\mathbf{Z}$ of correlated standard normals to produce a vector $\mathbf{U}$ of correlated uniforms over $(0,1)$ and then transforming each coordinate of $\mathbf{U}$ by the relevant inverse marginal. One approach to fitting requires, separately for each pair of coordinates of $\mathbf{X}$, the rank correlation, $r(\rho)$, or the product-moment correlation, $r_{\mathrm{L}}(\rho)$, where $\rho$ is the correlation of the corresponding coordinates of $\mathbf{Z}$, to equal some target $r^{*}$. We prove the existence and uniqueness of a solution for any feasible target, without imposing restrictions on the marginals. For the case where $r(\rho)$ cannot be computed exactly due to an infinite discrete support, the relevant infinite sums are approximated by truncation, and lower and upper bounds on the truncation errors are developed. With a function $\tilde{r}(\rho)$ defined by the truncated sums, a bound on the error $r\left(\rho^{*}\right)-r^{*}$ is given, where $\rho^{*}$ is a solution to $\tilde{r}\left(\rho^{*}\right)=r^{*}$. Based on this bound, an algorithm is proposed that determines truncation points so that the solution has any specified accuracy. The new truncation method has potential for significant work reduction relative to truncating heuristically, largely because as required accuracy decreases, so does the number of terms in the truncated sums. This is quantified with examples. The gain appears to increase with the heaviness of tails.

Keywords: statistics; multivariate distribution; unbounded discrete distribution; correlation; Gaussian copula.

History: Accepted by Marvin Nakayama, Area Editor for Simulation; received September 2011; revised October 2012, April 2013; accepted May 2013.
\end{abstract}




\section{Introduction}

A multivariate distribution may be specified via marginal univariate distributions and with dependence between marginals induced via a Gaussian (normal) copula. This is also known as the NORmal To Anything (NORTA) approach (Cario and Nelson, 1996, 1997). More precisely, let $F_{k}, k=1, \ldots, d$ be univariate (cumulative) distribution functions, write $\mathcal{N}_{\mathbf{R}}$ for the multivariate normal distribution with mean the zero vector and $d \times d$ correlation matrix $\mathbf{R}$, and construct $\mathbf{X}$ as

$$
\begin{aligned}
& \mathbf{Z}=\left(Z_{1}, \ldots Z_{d}\right) \sim \mathcal{N}_{\mathbf{R}} \\
& \mathbf{X}=\left(X_{1}, \ldots, X_{d}\right)=\left(F_{1}^{-1}\left[\Phi\left(Z_{1}\right)\right], \ldots, F_{d}^{-1}\left(\left[\Phi\left(Z_{d}\right)\right]\right)\right),
\end{aligned}
$$

where $\Phi$ is the standard normal distribution function (with mean 0 and variance 1 ) and $F_{k}^{-1}(u)=$ $\inf \left\{x: F_{k}(x) \geq u\right\}$ for $0<u<1$ is the inverse of $F_{k}$. By construction, the $k$-th marginal of $\mathbf{X}$ is $F_{k}$. Relative to other multivariate approaches, this model may be appealing by its separating the marginals from the dependence, which is contained in $\mathbf{R}$. The choice of Gaussian copula, while restrictive, facilitates fitting the model and sampling from it.

Consider the case $d=2$. The construction reduces to selecting the scalar correlation $\rho=$ $\operatorname{Corr}\left(Z_{1}, Z_{2}\right)$. One approach to specifying $\rho$ is to require that the rank correlation between $X_{1}$ and $X_{2}, r(\rho)=r\left(\rho ; F_{1}, F_{2}\right)=\operatorname{Corr}\left(F_{1}\left(X_{1}\right), F_{2}\left(X_{2}\right)\right)$, equals (matches) a target value $r^{*}$, which may be the sample rank correlation computed from data (observations of $\mathbf{X}$ ), or determined otherwise. This leads to the rank-correlation matching problem of solving

$$
r\left(\rho ; F_{1}, F_{2}\right)=r^{*}
$$

If $F_{1}$ and $F_{2}$ are both continuous (meaning absolutely continuous with respect to Lebesgue measure), then the rank-correlation matching problem is resolved by inversion of the formula $r(\rho)=$ $\operatorname{Corr}\left(\Phi\left(Z_{1}\right), \Phi\left(Z_{2}\right)\right)=(6 / \pi) \arcsin (\rho / 2)$ (Kruskal, 1958). An alternative approach seeks $\rho$ so that the product-moment correlation matches a target. Avramidis et al. (2009) have studied the discrete problem, where each marginal is discrete. Channouf and L'Ecuyer (2009) have studied the mixed problem, where one marginal is discrete and the other one is continuous. Correlation-matching is only one possible route to specifying a model with given marginals. Joe (2005) describes an alternative where marginals and the dependence parameter (of a general copula, not necessarily normal) are estimated in two separate phases, based on maximum-likelihood ideas.

The problem in dimension $d=2$ is central to Gaussian-copula-based constructions of random vectors in dimension $d>2$ and the VARTA class of stationary multivariate time series (Biller and Nelson, 2003). In these constructions, a correlation-matching problem is solved for certain pairs of coordinates. In the random-vector construction, a positive semi-definite matrix $\mathbf{R}$ is computed from the solutions of all coordinate pairs (Ghosh and Henderson, 2003). Channouf and L'Ecuyer (2012) use this methodology to model arrival counts in call centers over several periods of a day and find it most effective in fitting the full set of correlations. 
Our first contribution is a proof of existence and uniqueness of a solution for any feasible target, without imposing restrictions on the marginals. Intermediate results we obtain are expressions for the derivatives of the mean products $\mathbb{E}\left[X_{1} X_{2}\right]$ and $\mathbb{E}\left[F_{1}\left(X_{1}\right) F_{2}\left(X_{2}\right)\right]$ with respect to $\rho$; and that $r(\rho)$ and $r_{\mathrm{L}}(\rho)$ are differentiable and strictly increasing on $(-1,1)$.

Our second contribution is to approximate the function $r(\rho)$, with bounds on error, when it cannot be computed exactly due to an infinite (discrete) support, and to bound the error in induced correlation when solutions are computed via the approximation. An $X_{1}$ with infinite support gives rise to infinite sums in $\mathbb{E}\left[F_{1}\left(X_{1}\right)\right], \operatorname{Var}\left[F_{1}\left(X_{1}\right)\right]$, and $\mathbb{E}\left[F_{1}\left(X_{1}\right) F_{2}\left(X_{2}\right)\right]$. In the mean product, a doubly infinite sum arises if, additionally, $X_{2}$ is infinite. Avramidis et al. (2009) and Channouf and L'Ecuyer (2009) replace $r(\rho)$ by a version in which the relevant infinite sums are truncated. A simple heuristic is used there: truncate each infinite tail to the right at the quantile $x_{p}$ associated to a tail probability $p$ (quantile of order $1-p$ ), resulting in $x_{p}^{2}$ terms in the mean product. In this paper, the relevant infinite sums are approximated by truncation, and lower and upper bounds on the truncation errors are developed. With a function $\tilde{r}(\rho)$ defined by the truncated sums, a bound on the error $r\left(\rho^{*}\right)-r^{*}$ is given, where $\rho^{*}$ is a solution to $\tilde{r}\left(\rho^{*}\right)=r^{*}$ (the solution here is assumed to exist); such a problem can be (and is) solved as in Avramidis et al. (2009); Channouf and L'Ecuyer (2009). A simple algorithm is proposed that determines truncation points so that the solution has any required accuracy. Thus, we enable solving to desired accuracy, which is new.

Our focus on rank correlation is motivated by the fact that nonlinear dependence may be "missed" by product-moment correlation: Embrechts et al. (2002, Example 5) present a sequence of random vectors $(X, Y)$ that are comonotonic (or counter-monotonic), i.e., have a perfect positive (negative) dependence, and such that the product-moment correlation tends to zero; rank correlation, in contrast, captures the dependence. Separately, our bounding method does not apply to product-moment correlation.

Our approach may require less work than the heuristic, especially as the relevant quantile(s) become large. To quantify this point, consider the widely-used discrete Pareto family (Parulekar and Makowski, 1997; Suárez-González et al., 2002; Axtell, 2001; Deuchert and Brody, 2007). Defined for $\alpha>1$, and supported on the positive integers, the probability mass is $f(k)=k^{-\alpha} / \zeta(\alpha)$, where $\zeta(\alpha)=\sum_{k=1}^{\infty} k^{-\alpha}$ is Riemann's zeta function. For $X \sim \operatorname{Pareto}(\alpha)$, a simple calculation gives $x_{p} \sim((\alpha-1) \zeta(\alpha) p)^{1 /(1-\alpha)}$ as $p \rightarrow 0\left(a_{x} \sim b_{x}\right.$ means $\left.a_{x} / b_{x} \rightarrow 1\right)$. For $\alpha=2.1$ (finite mean, infinite variance) and $p=10^{-5}$, we have $x_{p}=21488$, and $x_{p}^{2}$ is large; in comparison, our method, with error tolerance $10^{-3}$ on $r^{*}$, requires about 35 thousand terms.

For the discrete problem, the truncation algorithm can be summarized as follows. The total error bound is the sum of a rightward error bound, which only depends on rightward truncation point(s), and a leftward error bound (that depends on leftward and rightward truncation points). We determine truncation points so that the total error bound is small enough as follows. First, we 
iteratively increase candidate rightward truncation points, increasing one of them by one at each iteration, until the rightward error bound is small enough. Then, we decrease candidate leftward truncation points, in similar fashion, until the leftward error bound is small enough. In the mixed problem, the algorithm is similar.

Although there is no simple way to specify equivalent tolerances between the heuristic and our approach, we nevertheless report numerical comparisons to help assess the potential for higher efficiency (work reduction). Comparing the heuristic with $p=10^{-6}$ to our method with tolerance $10^{-3}$ for Poisson, negative binomial, and Pareto marginals, we observe a consistent efficiency improvement, and far more pronounced with the Pareto heavier tails.

The remainder is organized as follows. Section 2 develops the existence and uniqueness results. The discrete problem is studied in Section 3; preliminary results have appeared in Avramidis (2009). The algorithm for determining truncation points is detailed in Section 3.5. The mixed problem is studied in Section 4. Numerical results appear in Section 5.

\section{General Marginals}

We assume throughout the paper that the marginals are non-degenerate. The rank correlation between $X_{1}$ and $X_{2}$ as in (1) is

$$
r(\rho)=\operatorname{Corr}\left(F_{1}\left(X_{1}\right), F_{2}\left(X_{2}\right)\right)=\frac{g(\rho)-\mu_{1} \mu_{2}}{\sigma_{1} \sigma_{2}},
$$

where $\mu_{k}=\mathbb{E}\left[F_{k}\left(X_{k}\right)\right] ; \sigma_{k}^{2}=\operatorname{Var}\left[F_{k}\left(X_{k}\right)\right] ;$ and

$$
g(\rho)=\mathbb{E}\left[F_{1}\left(X_{1}\right) F_{2}\left(X_{2}\right)\right]=\int_{0}^{1} \int_{0}^{1} \mathbb{P}\left(h_{1}\left(Z_{1}\right)>x, h_{2}\left(Z_{2}\right)>y\right) \mathrm{d} x \mathrm{~d} y
$$

where $h_{k}=F_{k} \circ F_{k}^{-1} \circ \Phi$ (the composite function). The last equality is based on the fact that for any random variables $X$ and $Y$,

$$
\mathbb{E}[X Y]=\int_{-\infty}^{\infty} \int_{-\infty}^{\infty} \mathbb{P}(X>x, Y>y) \mathrm{d} x \mathrm{~d} y
$$

provided the expectation is finite (e.g. Lehmann, 1966, Lemma 2).

We now represent $g$ and its derivative with respect to $\rho$ as integrals involving the bivariate normal density. We need a (generalized) inverse of the functions $h_{k}$. To this end, let $F$ be a (cumulative) distribution function (c.d.f. in short), let $D_{F}$ be the set of discontinuity points of $F$, and put $G(F)=\cup_{x \in D_{F}}(F(x-), F(x))$; this is the set of $u$ for which there exists no $v$ such that $F(v)=u$, due to discontinuity of $F$. The inverse of $F$ is $F^{-1}(u)=\inf \{x: F(x) \geq u\}$. We now define the inverse of $F \circ F^{-1}$ as

$$
\left(F \circ F^{-1}\right)^{-1}(u)= \begin{cases}F\left(F^{-1}(u)-\right) & u \in G(F) \\ v & \text { otherwise }\end{cases}
$$


for $u \in(0,1)$, where $F\left(F^{-1}(u)-\right)$ is the left limit of $F$ at $F^{-1}(u)$. A special case of $(6)$ that we need later has a discrete $F$ with cumulative probabilities $0=f_{0}<f_{1}<\ldots$; then $\left(F \circ F^{-1}\right)^{-1}(u)=f_{i-1}$ whenever $u \in\left(f_{i-1}, f_{i}\right]$. Now define $h_{k}^{-1}=\Phi^{-1} \circ\left(F_{k} \circ F_{k}^{-1}\right)^{-1}$, where $\Phi^{-1}$ is the inverse of $\Phi$. For any $F$, one may verify that

$$
F \circ F^{-1}(v)>u \Longleftrightarrow v>\left(F \circ F^{-1}\right)^{-1}(u)
$$

and thus (4) gives

$$
g(\rho)=\int_{0}^{1} \int_{0}^{1} \mathbb{P}\left(Z_{1}>h_{1}^{-1}(x), Z_{2}>h_{2}^{-1}(y)\right) \mathrm{d} x \mathrm{~d} y=\int_{0}^{1} \int_{0}^{1} \bar{\Phi}_{\rho}\left(h_{1}^{-1}(x), h_{2}^{-1}(y)\right) \mathrm{d} x \mathrm{~d} y,
$$

where $\bar{\Phi}_{\rho}(x, y)=\int_{x}^{\infty} \int_{y}^{\infty} \phi_{\rho}(z, w) \mathrm{d} z \mathrm{~d} w$, where $\phi_{\rho}(x, y)$ is the density at $(x, y)$ of the bivariate standard normal distribution with correlation $\rho$. Certain invariance properties of rank correlation can now be seen. First, if $F_{k}$ is continuous, then $h_{k}^{-1}()=\Phi^{-1}()$, and then (8) shows that the dependence on $F_{k}$ disappears. In particular, for $F_{1}$ discrete and $F_{2}$ continuous, $r\left(\rho ; F_{1}, F_{2}\right)$ is a function of $\rho$ and $F_{1}$ only. Second, the locations of any discontinuity points do not matter (to $g$ and $r$ ) - only the values attained by the c.d.f. do. This property is inherited from the inverse $\left(F \circ F^{-1}\right)^{-1}$ defined above. Differentiation of (8) gives

$$
\frac{\mathrm{d}}{\mathrm{d} \rho} g(\rho)=\int_{0}^{1} \int_{0}^{1} \frac{\mathrm{d}}{\mathrm{d} \rho} \bar{\Phi}_{\rho}\left(h_{1}^{-1}(x), h_{2}^{-1}(y)\right) \mathrm{d} x \mathrm{~d} y=\int_{0}^{1} \int_{0}^{1} \phi_{\rho}\left(h_{1}^{-1}(x), h_{2}^{-1}(y)\right) \mathrm{d} x \mathrm{~d} y, \quad \rho \in(-1,1) .
$$

The derivative can pass inside the integral by an argument as in the proof of Theorem 9.42 in Rudin (1976) on noting that $\phi_{\rho}\left(h_{1}^{-1}(x), h_{2}^{-1}(y)\right)$ has a bounded gradient with respect to $(\rho, x, y)$ almost everywhere on $(-1,1) \times \mathbb{R}^{2}$. We then use that $(\mathrm{d} / \mathrm{d} \rho) \bar{\Phi}_{\rho}(x, y)=\phi_{\rho}(x, y)$ (e.g. Avramidis et al., 2009, eq. (13)).

An analogous development for the product-moment correlation follows: $r_{\mathrm{L}}(\rho)=\operatorname{Corr}\left(X_{1}, X_{2}\right)=$ $\left(g_{\mathrm{L}}(\rho)-\mathbb{E}\left[X_{1}\right] \mathbb{E}\left[X_{2}\right]\right) / \sqrt{\operatorname{Var}\left(X_{1}\right) \operatorname{Var}\left(X_{2}\right)}$, where, using (5),

$$
\begin{aligned}
g_{\mathrm{L}}(\rho)=\mathbb{E}\left[X_{1} X_{2}\right] & =\int_{-\infty}^{\infty} \int_{-\infty}^{\infty} \mathbb{P}\left(F_{1}^{-1}\left(\Phi\left(Z_{1}\right)\right)>x, F_{2}^{-1}\left(\Phi\left(Z_{2}\right)\right)>y\right) \mathrm{d} x \mathrm{~d} y \\
& =\int_{-\infty}^{\infty} \int_{-\infty}^{\infty} \mathbb{P}\left(Z_{1}>\Phi^{-1}\left(F_{1}(x)\right), Z_{2}>\Phi^{-1}\left(F_{2}(y)\right)\right) \mathrm{d} x \mathrm{~d} y \\
& =\int_{-\infty}^{\infty} \int_{-\infty}^{\infty} \bar{\Phi}_{\rho}\left(\Phi^{-1}\left(F_{1}(x)\right), \Phi^{-1}\left(F_{2}(y)\right)\right) \mathrm{d} x \mathrm{~d} y .
\end{aligned}
$$

We used above the equivalence $F^{-1}(u)>x \Longleftrightarrow u>F(x)$, valid for any c.d.f. $F$ and $u \in(0,1)$ (Asmussen and Glynn, 2007, Proposition 2.2(a), page 38). Differentiation of (10) gives

$$
\begin{aligned}
\frac{\mathrm{d}}{\mathrm{d} \rho} g_{\mathrm{L}}(\rho) & =\int_{-\infty}^{\infty} \int_{-\infty}^{\infty} \frac{\mathrm{d}}{\mathrm{d} \rho} \bar{\Phi}_{\rho}\left(\left(\Phi^{-1}\left(F_{1}(x)\right), \Phi^{-1}\left(F_{2}(y)\right) \mathrm{d} x \mathrm{~d} y\right.\right. \\
& =\int_{-\infty}^{\infty} \int_{-\infty}^{\infty} \phi_{\rho}\left(\Phi^{-1}\left(F_{1}(x)\right), \Phi^{-1}\left(F_{2}(y)\right)\right) \mathrm{d} x \mathrm{~d} y, \quad \rho \in(-1,1) .
\end{aligned}
$$


The derivative can pass inside the integral because $\phi_{\rho}\left(\Phi^{-1}\left(F_{1}(x)\right), \Phi^{-1}\left(F_{2}(y)\right)\right)$ has a bounded gradient with respect to $(\rho, x, y)$ almost everywhere on $(-1,1) \times \mathbb{R}^{2}$. Thus:

Proposition 1 Let $F_{1}$ and $F_{2}$ be c.d.f.'s of non-degenerate distributions. Put $X_{k}=F_{k}^{-1}\left(\Phi\left(Z_{k}\right)\right)$ for $k=1,2$, where $\left(Z_{1}, Z_{2}\right)$ is bivariate normal with standard-normal marginals and correlation $\rho$. The function $g(\rho)$ in (4) has derivative (9), and the function $g_{\mathrm{L}}(\rho)$ in (10) has derivative (11).

The differentiability of the function $g$ implies its continuity, and the same properties hold for function $r$. Moreover, continuity at the endpoints, -1 and 1, follows by Cario and Nelson (1996, Theorem 2). Then, the Intermediate Value Theorem implies that for any $r^{*} \in[r(-1), r(1)]$, the equation $r(\rho)=r^{*}$ has a solution. Likewise, the differentiability of $g_{\mathrm{L}}$ on $(-1,1)$ implies that for any $\delta>0, g_{\mathrm{L}}$ and $r_{\mathrm{L}}$ are continuous on $[-1+\delta, 1-\delta]$ (continuity at -1 and 1 may require extra conditions), proving the solution's existence for $r^{*} \in\left[r_{\mathrm{L}}(-1+\delta), r_{\mathrm{L}}(1-\delta)\right]$. Moreover, the solution in each case is unique, as each of $r$ and $r_{\mathrm{L}}$ is strictly increasing in $\rho$ (the integrand in each of (9) and (11) is positive on a set of positive Lebesgue measure and non-negative everywhere). Thus, the existence and uniqueness results in Cario and Nelson (1996, Theorem 1) and Avramidis et al. (2009) extend, without any restrictions on the marginals:

Corollary 1 The functions $g$ and $g_{\mathrm{L}}$ are differentiable and strictly increasing everywhere. For any $r^{*} \in[r(-1), r(1)]$, the equation $r(\rho)=r^{*}$ has a unique solution. For any $\delta>0$ and for $r^{*} \in\left[r_{\mathrm{L}}(-1+\delta), r_{\mathrm{L}}(1-\delta)\right]$, the equation $r_{\mathrm{L}}(\rho)=r^{*}$ has a unique solution.

\section{The Discrete Problem}

\subsection{Preliminaries}

The discrete rank-correlation-matching problem refers to solving $r(\rho)=r^{*}$ where the marginals $F_{1}$ and $F_{2}$ are both discrete. For simplicity, we assume the marginals have an infinite tail to the right only. We enumerate the support points after putting them in increasing order as $\{0,1,2, \ldots\}$. For the $k$-th marginal, $p_{k, i}$ denotes the probability mass at $i$; we put $f_{k, i}=\sum_{j=0}^{i} p_{k, j}$ and $f_{k,-1}=0$. As stated following (6), $\left(F_{k} \circ F_{k}^{-1}\right)^{-1}(v)=f_{k, i-1}$ for all $v \in\left(f_{k, i-1}, f_{k, i}\right]$. Then (8) gives

$$
g(\rho)=\sum_{i=0}^{\infty} p_{1, i} \sum_{j=0}^{\infty} p_{2, j} \bar{\Phi}_{\rho}\left(z_{1, i-1}, z_{2, j-1}\right),
$$

where $z_{k, i}=\Phi^{-1}\left(f_{k, i}\right)$ and $z_{k,-1}=-\infty$. This is equation (10) of Avramidis et al. (2009), seen here to be a special case of $(8)$.

\subsection{Approximation of the Mean and the Variance}

The task is to approximate the means and variances in (3). To lighten notation, we work with a single marginal and later apply the forthcoming results to each marginal. Denote $p_{i}$ the probability 
mass at $i$ and $f_{i}=\sum_{j=0}^{i} p_{j}$ the cumulative probability at $i$. We will approximate the mean $\mu=\mathbb{E}[F(X)]=\sum_{i=0}^{\infty} f_{i} p_{i}$ and the variance $\sigma^{2}=\sum_{i=0}^{\infty} f_{i}^{2} p_{i}-\mu^{2}$. Note that $\mu<1$ and $\sigma^{2}>0$, by non-degeneracy. The approximation is via the corresponding exact moments of the finite-support random variable, $X_{n}$, obtained by shifting to the point $n+1$ the probability mass of all the points to its right, so that the resulting mass at $n+1$ is the tail probability $t_{n}=1-f_{n}=\sum_{i>n} p_{i}$. With $F_{n}$ denoting the c.d.f. of $X_{n}$, the approximate mean is

$$
\tilde{\mu}_{n}=\mathbb{E}\left[F_{n}\left(X_{n}\right)\right]=\sum_{i=0}^{n} f_{i} p_{i}+1-f_{n}
$$

and the approximate variance is

$$
\tilde{\sigma}_{n}^{2}=\operatorname{Var}\left[F_{n}\left(X_{n}\right)\right]=\tilde{\mu}_{n}^{(2)}-\tilde{\mu}_{n}^{2}
$$

where $\tilde{\mu}_{n}^{(2)}=\mathbb{E}\left[F_{n}^{2}\left(X_{n}\right)\right]=\sum_{i=0}^{n} f_{i}^{2} p_{i}+1-f_{n}$.

We now derive sequences that bound $\mu$ and $\sigma^{2}$ from below and above and that converge to these targets in each case. We define $x^{+}=\max (x, 0)$.

Lemma 1 (i) (Sequences bounding $\mu$ below and above and converging to it.) Define $\underline{\mu}_{n}=\left(\tilde{\mu}_{n}-\right.$ $\left.t_{n} t_{n+1}\right)^{+}$. We have

$$
\underline{\mu}_{n} \leq \mu \leq \tilde{\mu}_{n} \text { for all } n
$$

and $\tilde{\mu}_{n} \downarrow \mu$ and $\underline{\mu}_{n} \rightarrow \mu$ as $n \rightarrow \infty$.

(ii) (Sequences bounding $\sigma^{2}$ below and above and converging to it.) Define

$$
\underline{\sigma}_{n}^{2}=\tilde{\sigma}_{n}^{2}-2\left(1-\underline{\mu}_{n}\right) t_{n} t_{n+1} \quad \text { and } \quad \bar{\sigma}_{n}^{2}= \begin{cases}\tilde{\sigma}_{n}^{2}-l_{n} & n<n^{*} \\ \tilde{\sigma}_{n}^{2} & n \geq n^{*}\end{cases}
$$

where $l_{n}=\left(1+f_{n}-\tilde{\mu}_{n-1}-\tilde{\mu}_{n}\right) t_{n} t_{n+1}$ and $n^{*}=\min \left\{n: 1+f_{n}-\tilde{\mu}_{n-1}-\tilde{\mu}_{n}>0\right\}<\infty$. We have

$$
\underline{\sigma}_{n}^{2} \leq \sigma^{2} \leq \bar{\sigma}_{n}^{2} \text { for all } n
$$

and $\left\{\tilde{\sigma}_{n}^{2}\right\}_{n=n^{*}}^{\infty} \downarrow \sigma^{2}$ and $\underline{\sigma}_{n}^{2} \rightarrow \sigma^{2}$ as $n \rightarrow \infty$.

Proof. Part (i). Write

$$
\tilde{\mu}_{i-1}-\tilde{\mu}_{i}=\sum_{k \leq i-1} f_{k} p_{k}+1-f_{i-1}-\sum_{k \leq i} f_{k} p_{k}-\left(1-f_{i}\right)=p_{i}\left(1-f_{i}\right)>0
$$

and $\tilde{\mu}_{n}-\mu=\sum_{i>n}\left(\tilde{\mu}_{i-1}-\tilde{\mu}_{i}\right)$, by the nonnegativity of the summands. Thus

$$
0<\tilde{\mu}_{n}-\mu=\sum_{i>n}\left(\tilde{\mu}_{i-1}-\tilde{\mu}_{i}\right)=\sum_{i>n} p_{i}\left(1-f_{i}\right) \leq\left(1-f_{n+1}\right)\left(1-f_{n}\right)=t_{n+1} t_{n} .
$$

The assertion $\lim \underline{\mu}_{n}=\mu$ follows from $\lim _{n \rightarrow \infty} t_{n} t_{n+1}=0$. 
Part (ii). We have

$$
\begin{aligned}
\tilde{\sigma}_{i-1}^{2}-\tilde{\sigma}_{i}^{2} & =\tilde{\mu}_{i-1}^{(2)}-\tilde{\mu}_{i}^{(2)}-\left(\tilde{\mu}_{i-1}^{2}-\tilde{\mu}_{i}^{2}\right) \\
& =\sum_{k \leq i-1} f_{k}^{2} p_{k}+1-f_{i-1}-\sum_{k \leq i} f_{k}^{2} p_{k}-\left(1-f_{i}\right)-\left(\tilde{\mu}_{i-1}-\tilde{\mu}_{i}\right)\left(\tilde{\mu}_{i-1}+\tilde{\mu}_{i}\right) \\
& =p_{i}\left(1-f_{i}^{2}\right)-p_{i}\left(1-f_{i}\right)\left(\tilde{\mu}_{i-1}+\tilde{\mu}_{i}\right) \\
& =p_{i}\left(1-f_{i}\right)\left(1+f_{i}-\tilde{\mu}_{i-1}-\tilde{\mu}_{i}\right)
\end{aligned}
$$

by using (16) in the third step. The above is nonnegative for all $i$ large enough, so

$$
\tilde{\sigma}_{n}^{2}-\sigma^{2}=\sum_{i>n}\left(\tilde{\sigma}_{i-1}^{2}-\tilde{\sigma}_{i}^{2}\right)
$$

We claim that

$$
2\left(1-\underline{\mu}_{n}\right) t_{n} t_{n+1} \geq \sum_{i>n} p_{i}\left(1-f_{i}\right)\left(1+f_{i}-\tilde{\mu}_{i-1}-\tilde{\mu}_{i}\right) \geq \begin{cases}\left(1+f_{n}-\tilde{\mu}_{n-1}-\tilde{\mu}_{n}\right) t_{n} t_{n+1}, & n<n^{*} \\ 0, & n \geq n^{*}\end{cases}
$$

and observe that the quantity in the middle is $\tilde{\sigma}_{n}^{2}-\sigma^{2}$; then a simple rearrangement will prove (15). It remains to prove (20). Note that the sequence $\left\{1+f_{i}-\tilde{\mu}_{i-1}-\tilde{\mu}_{i}\right\}_{i=1}^{\infty}$ is monotonically increasing to $2(1-\mu)$ (since $\left\{f_{i}\right\}_{i=0}^{\infty} \uparrow 1$ and $\left.\left\{\tilde{\mu}_{i}\right\}_{i=0}^{\infty} \downarrow \mu\right)$, so

$$
2(1-\mu) \sum_{i>n} p_{i}\left(1-f_{i}\right) \geq \sum_{i>n}\left(1+f_{i}-\tilde{\mu}_{i-1}-\tilde{\mu}_{i}\right) p_{i}\left(1-f_{i}\right) \geq\left(1+f_{n}-\tilde{\mu}_{n-1}-\tilde{\mu}_{n}\right) \sum_{i>n} p_{i}\left(1-f_{i}\right)
$$

In the above, we may substitute looser bounds, as follows. The upper bound (left side) is positive, so we may substitute for $\sum_{i>n} p_{i}\left(1-f_{i}\right)$ and $1-\mu$ the respective upper bounds $t_{n} t_{n+1}$ and $1-\underline{\mu}_{n}$. The lower bound (right side) is negative (positive) when $n<n^{*}\left(n \geq n^{*}\right)$ respectively; in the negative case, we may substitute for $\sum_{i>n} p_{i}\left(1-f_{i}\right)$ the upper bound $t_{n} t_{n+1}$; in the positive case, we may substitute zero. These substitutions give (20), and this completes the proof of (15). The assertion $\left\{\tilde{\sigma}_{n}^{2}\right\}_{n=n^{*}}^{\infty} \downarrow \sigma^{2}$ holds on noting that the sequence $\left\{1+f_{i}-\tilde{\mu}_{i-1}-\tilde{\mu}_{i}\right\}_{i=0}^{\infty}$ is monotonically increasing and its $n^{*}$-th term is positive, so each summand in (19) is positive for $n \geq n^{*}$. The assertion $\lim \underline{\sigma}_{n}^{2}=\sigma^{2}$ follows from $\lim _{n \rightarrow \infty} t_{n} t_{n+1}=0$.

Results (14) and (15) hold at any $n$, so truncation of a finite support (a special case of an infinite one) is also covered. In view of $\lim \underline{\sigma}_{n}^{2}=\sigma^{2}>0$, we may define for $n$ large enough the real number $\underline{\sigma}_{n}=\sqrt{\underline{\sigma}_{n}^{2}}$.

\subsection{Approximation of the Mean Product}

For a vector $\mathbf{n}=\left(l_{1}, r_{1}, l_{2}, r_{2}\right)$, define the approximation $g_{\mathbf{n}}(\rho)$ of $g(\rho)$ as the right side of (12) truncated so the range of $i$ is restricted to $l_{1} \leq i \leq r_{1}$ and the range of $j$ is restricted to $l_{2} \leq j \leq r_{2}$. 
Lemma 2 We have

$$
0 \leq g(\rho)-g_{\mathbf{n}}(\rho) \leq \sum_{k=1}^{2}\left(f_{k, l_{k}-1}+t_{k, r_{k}}^{2}\right) \quad \text { for all } \rho .
$$

Proof. By the non-negativity of each summand in (12), we have, for any $\rho$,

$$
\begin{aligned}
0 \leq g(\rho)-g_{\mathbf{n}}(\rho) \leq & \sum_{i<l_{1}} p_{1, i} \sum_{j=0}^{\infty} p_{2, j} \bar{\Phi}_{\rho}\left(z_{1, i-1}, z_{2, j-1}\right)+\sum_{i>r_{1}} p_{1, i} \sum_{j=0}^{\infty} p_{2, j} \bar{\Phi}_{\rho}\left(z_{1, i-1}, z_{2, j-1}\right) \\
& +\sum_{j<l_{2}} p_{2, j} \sum_{i=0}^{\infty} p_{1, i} \bar{\Phi}_{\rho}\left(z_{1, i-1}, z_{2, j-1}\right)+\sum_{j>r_{2}} p_{2, j} \sum_{i=0}^{\infty} p_{1, i} \bar{\Phi}_{\rho}\left(z_{1, i-1}, z_{2, j-1}\right) .(23)
\end{aligned}
$$

Since $\bar{\Phi}_{\rho}(x, y)$ is non-decreasing in $\rho$, we have

$$
\bar{\Phi}_{\rho}(x, y) \leq \bar{\Phi}_{1}(x, y)=\bar{\Phi}(\max (x, y))=\min (\bar{\Phi}(x), \bar{\Phi}(y)) \quad \text { for all } \rho
$$

where $\bar{\Phi}=1-\Phi$ is the standard univariate normal complementary c.d.f.. Using this, an upper bound for the first of the four terms on the right in (23) is

$$
\sum_{i<l_{1}} p_{1, i} \bar{\Phi}\left(z_{1, i-1}\right) \sum_{j=0}^{\infty} p_{2, j}=\sum_{i<l_{1}} p_{1, i} t_{1, i-1} \leq \sum_{i<l_{1}} p_{1, i}=f_{1, l_{1}-1}
$$

upon noting that $\bar{\Phi}\left(z_{1, i-1}\right)=t_{1, i-1}$ and $\sum_{j=0}^{\infty} p_{2, j}=1$; and an upper bound for the second term on the right of $(23)$ is

$$
\sum_{i>r_{1}} p_{1, i} \bar{\Phi}\left(z_{1, i-1}\right) \sum_{j=0}^{\infty} p_{2, j}=\sum_{i>r_{1}} p_{1, i} t_{1, i-1} \leq t_{1, r_{1}} t_{1, r_{1}+1} .
$$

The bounds (25) and (26) and their analogs for the third and fourth term in (23) give (22).

\subsection{Approximation of the Rank Correlation}

For $k \in\{1,2\}$, and for the purpose of approximating $\mu_{k}$ and $\sigma_{k}$, we truncate marginal $k$ to the right of $r_{k}$, as described in Section 3.2. We will approximate the function $r(\rho)$ as $\tilde{r}_{\mathbf{n}}(\rho)=$ $\left(g_{\mathbf{n}}(\rho)-\tilde{\mu}_{1, r_{1}} \tilde{\mu}_{2, r_{2}}\right) /\left(\tilde{\sigma}_{1, r_{1}} \tilde{\sigma}_{2, r_{2}}\right)$, where $\mathbf{n}=\left(l_{1}, r_{1}, l_{2}, r_{2}\right)$ gives the truncation of the sum about $g_{\mathbf{n}}$, as in Section 3.3, and the sums $\mu_{k}$ and $\sigma_{k}$ are truncated to the right only. Left-truncation of these sums would complicate the error analysis while having little impact on computing cost.

Observe that $\tilde{r}_{\mathbf{n}}$ is a continuous strictly increasing function on $[-1,1]$, and thus has an inverse; that is, for $r^{*} \in\left[\tilde{r}_{\mathbf{n}}(-1), \tilde{r}_{\mathbf{n}}(1)\right]$, there exists a unique $\rho$ such that $\tilde{r}_{\mathbf{n}}(\rho)=r^{*}$, which we denote $\tilde{r}_{\mathbf{n}}^{-1}\left(r^{*}\right)$. This follows immediately from Corollary 1 by observing that $g_{\mathbf{n}}$ is the $g$ in (12) corresponding to the finite support that results when for each $k \in\{1,2\}$ we shift to the point $r_{k}$ the probability mass of the points to its right and we shift to the point $l_{k}$ the probability mass of the points to its left. Our main result is as follows. 
Proposition 2 Let $\rho^{*}=\tilde{r}_{\mathbf{n}}^{-1}\left(r^{*}\right)$, where $r^{*} \in\left[\tilde{r}_{\mathbf{n}}(-1), \tilde{r}_{\mathbf{n}}(1)\right]$. Provided that $\underline{\sigma}_{1}^{2}$ and $\underline{\sigma}_{2}^{2}$ are positive, we have

$$
\zeta_{\mathbf{n}} \leq r\left(\rho^{*}\right)-r^{*} \leq \eta_{\mathbf{n}}+\theta_{\mathbf{n}} \text { for all } n
$$

where

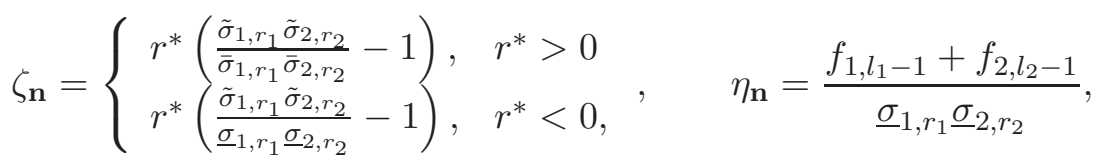

and

$$
\theta_{\mathbf{n}}= \begin{cases}\frac{t_{1, r_{1}}^{2}+t_{2, r_{2}}^{2}+\tilde{\mu}_{1, r_{1}} \tilde{\mu}_{2, r_{2}}-\underline{\mu}_{1, r_{1}} \underline{\mu}_{2, r_{2}}}{\underline{\sigma}_{1, r_{1}} \underline{\sigma}_{2, r_{2}}}+r^{*}\left(\frac{\tilde{\sigma}_{1, r_{1}} \tilde{\sigma}_{2, r_{2}}}{\underline{\sigma}_{1, r_{1}} \underline{\sigma}_{2, r_{2}}}-1\right), & r^{*}>0 \\ \frac{t_{1, r_{1}}^{2}+t_{2, r_{2}}^{2}+\tilde{\mu}_{1, r_{1}} \tilde{\mu}_{2, r_{2}}-\underline{\mu}_{1, r_{1}} \underline{\mu}_{2, r_{2}}}{\underline{\sigma}_{1, r_{1}} \underline{\sigma}_{2, r_{2}}}+r^{*}\left(\frac{\tilde{\sigma}_{1, r_{1}} \tilde{\sigma}_{2, r_{2}}}{\bar{\sigma}_{1, r_{1}} \bar{\sigma}_{2, r_{2}}}-1\right), & r^{*}<0 .\end{cases}
$$

Proof. Putting $\tilde{h}_{\mathbf{n}}(\rho)=g_{\mathbf{n}}(\rho)-\tilde{\mu}_{1, r_{1}} \tilde{\mu}_{2, r_{2}}-r^{*} \tilde{\sigma}_{1, r_{1}} \tilde{\sigma}_{2, r_{2}}$, we have $\tilde{h}_{\mathbf{n}}\left(\rho^{*}\right)=0$ and

$$
r\left(\rho^{*}\right)-r^{*}=\frac{g\left(\rho^{*}\right)-g_{\mathbf{n}}\left(\rho^{*}\right)+\tilde{h}_{\mathbf{n}}\left(\rho^{*}\right)+\tilde{\mu}_{1, r_{1}} \tilde{\mu}_{2, r_{2}}-\mu_{1} \mu_{2}+r^{*}\left(\tilde{\sigma}_{1, r_{1}} \tilde{\sigma}_{2, r_{2}}-\sigma_{1} \sigma_{2}\right)}{\sigma_{1} \sigma_{2}} .
$$

Now (27) follows from the bounds on $g(\rho)-g_{\mathbf{n}}(\rho)$ in (22); the bounds on $\mu_{k}$ as in (14); and the bounds on $\sigma_{k}^{2}$ as in (15).

We observe, even though we never use, that the set $\left\{\rho: \operatorname{sign}(\rho) \neq \operatorname{sign}\left(\tilde{r}_{\mathbf{n}}(\rho)\right)\right\}$ is the interval between zero and $\tilde{r}_{\mathbf{n}}^{-1}(0)$, and that this interval can be made arbitrarily small: for $l_{1}=l_{2}=0$, we can show that $\lim _{r_{1}, r_{2} \rightarrow \infty} \tilde{r}_{\mathbf{n}}^{-1}(0)=0$.

Note that $\zeta_{\mathbf{n}} \leq 0$ and $\eta_{\mathbf{n}}, \theta_{\mathbf{n}} \geq 0$. We now derive asymptotic relations about the error bounds under the assumption $l_{1}=l_{2}=0$ and $r_{1}, r_{2} \rightarrow \infty$. To lighten the notation here, put $\tilde{\mu}_{k}=$ $\tilde{\mu}_{k, r_{k}}, \underline{\mu}_{k}=\underline{\mu}_{k, r_{k}}, \tilde{\sigma}_{k}=\tilde{\sigma}_{k, r_{k}}, \underline{\sigma}_{k}=\underline{\sigma}_{k, r_{k}}, \bar{\sigma}_{k}=\bar{\sigma}_{k, r_{k}}$. Now observe: (a) for all $r_{k}$ large enough, we have $\underline{\mu}_{k}=\tilde{\mu}_{k}-t_{k, r_{k}} t_{k, r_{k}+1}$, so $\tilde{\mu}_{1} \tilde{\mu}_{2}-\underline{\mu}_{1} \underline{\mu}_{2}=\mu_{2} t_{1, r_{1}}^{2}+\mu_{1} t_{2, r_{2}}^{2}+o\left(t_{1, r_{1}}^{2}+t_{2, r_{2}}^{2}\right)$, where $o(\cdot)$ has the usual meaning; (b) from $\underline{\sigma}_{k}=\tilde{\sigma}_{k} \sqrt{1-2\left(1-\underline{\mu}_{k}\right) t_{k, r_{k}} t_{k, r_{k}+1} / \tilde{\sigma}_{k}^{2}}$ and the Taylor expansion $\sqrt{1-x}=1-x / 2+o(x)$ as $x \downarrow 0$, we obtain $\underline{\sigma}_{k}=\tilde{\sigma}_{k}\left[1-\left(1-\mu_{k}\right) t_{k, r_{k}}^{2} / \sigma_{k}^{2}\right]+o\left(t_{k, r_{k}}^{2}\right)$; then a simple calculation gives $\tilde{\sigma}_{1} \tilde{\sigma}_{2} / \underline{\sigma}_{1} \underline{\sigma}_{2}-1=a_{1} t_{1, r_{1}}^{2}+a_{2} t_{2, r_{2}}^{2}+o\left(t_{1, r_{1}}^{2}+t_{2, r_{2}}^{2}\right)$, where $a_{k}=\left(1-\mu_{k}\right) / \sigma_{k}^{2}$; and (c) $\tilde{\sigma}_{1} \tilde{\sigma}_{2} /\left(\bar{\sigma}_{1} \bar{\sigma}_{2}\right)=1$, provided $r_{k} \geq n_{k}^{*}$, the $n_{k}^{*}$ being as in Lemma 1 . From these observations, we obtain: (i) when $r^{*}<0$, we have $\zeta_{\mathbf{n}}=r^{*}\left(a_{1} t_{1, r_{1}}^{2}+a_{2} t_{2, r_{2}}^{2}\right)+o\left(t_{1, r_{1}}^{2}+t_{2, r_{2}}^{2}\right)$; when $r^{*}>0$, we have $\zeta_{\mathbf{n}}=0$, provided $r_{k} \geq n_{k}^{*}$; and (ii) $\theta_{\mathbf{n}}=b_{1} t_{1, r_{1}}^{2}+b_{2} t_{2, r_{2}}^{2}+o\left(t_{1, r_{1}}^{2}+t_{2, r_{2}}^{2}\right)$, where we define $b_{k}=\left(1+\mu_{3-k}\right) /\left(\sigma_{1} \sigma_{2}\right)+r^{*} a_{k}$ for $r^{*}>0$ and $b_{k}=\left(1+\mu_{3-k}\right) /\left(\sigma_{1} \sigma_{2}\right)$ for $r^{*}<0$.

Remark 1 Write $c$ for the right side of (22). It is not difficult to see that

$$
\left.\begin{array}{ll}
\frac{g_{\mathbf{n}}(\rho)-\tilde{\mu}_{1, r_{1}} \tilde{\mu}_{2, r_{2}}}{\bar{\sigma}_{1, r_{1}} \bar{\sigma}_{2, r_{2}}} \leq \frac{g(\rho)-\mu_{1} \mu_{2}}{\sigma_{1} \sigma_{2}} \leq \frac{g_{\mathbf{n}}(\rho)+c-\underline{\mu}_{1, r_{1}} \underline{\mu}_{2, r_{2}}}{\underline{\sigma}_{1, r_{1}} \underline{\sigma}_{2, r_{2}}}, & \rho>0 \\
\frac{g_{\mathbf{n}}(\rho)-\tilde{\mu}_{1, r_{1}} \tilde{\mu}_{2, r_{2}}}{\underline{\sigma}_{1, r_{1}} \underline{\sigma}_{2, r_{2}}} \leq \frac{g(\rho)-\mu_{1} \mu_{2}}{\sigma_{1} \sigma_{2}} \leq \frac{g_{\mathbf{n}}(\rho)+c-\underline{\mu}_{1, r_{1}} \underline{\mu}_{2, r_{2}}}{\bar{\sigma}_{1, r_{1}} \bar{\sigma}_{2, r_{2}}}, & \rho<0
\end{array}\right\}
$$


The distance between the lower and upper bounds above converges to zero when $l_{1}=l_{2}=0$ and $r_{1}, r_{2} \rightarrow \infty$ (by results (a) to (c) after Proposition 2). Thus, (29) may be used to compute $r(\rho)$ for any $\rho$, including the extreme correlations $r(-1)$ and $r(1)$, to any desired accuracy.

\subsection{Truncation Algorithm}

The work to compute the root of $\tilde{r}_{\mathbf{n}}(\rho)=r^{*}$ can be expected to be roughly linear in $w=\left(r_{1}-l_{1}+\right.$ $1)\left(r_{2}-l_{2}+1\right)$. This is because $w$ bivariate normal integrals are involved in evaluating $g_{\mathbf{n}}(\rho)$ at any candidate; if derivatives are to be used (Avramidis et al., 2009), then $w$ derivatives, one for each term of (12), are involved at any candidate; and empirical results in Avramidis et al. (2009) are consistent with our claim. Then, accuracy and efficiency considerations suggest that $\mathbf{n}$ be chosen to minimize $w$ subject to the error bounds in (27) being within given limits.

Rather than solving such a minimization problem exactly, we propose Algorithm 1 below. This is an approximate algorithm, and it is designed for simplicity and not efficiency because the ensuing root-finding work is far more demanding, as numerical results will show. First we reduce the quantity $\max \left(-\zeta_{\mathbf{n}}, \theta_{\mathbf{n}}\right)$ — called the rightward error bound, as it only depends on the $r_{k}$-as follows: we initialize $r_{1}$ and $r_{2}$ as the smallest support point, 0 and iteratively increase $r_{1}$ or $r_{2}$ by one, choosing for simplicity the one that corresponds to the larger tail probability to the right, $t_{k, r_{k}}$, until both lower bounds on variance $\left(\underline{\sigma}_{k, r_{k}}^{2}\right)$ are positive and the rightward error bound is no larger than $\delta_{\mathrm{r}}$, where $\delta_{\mathrm{r}}>0$ is a specified tolerance. Having determined $r_{1}$ and $r_{2}$, we then reduce the quantity $\eta_{\mathbf{n}}$ —called the leftward error bound because the $r_{k}$ have been fixed - as follows: we initialize $l_{k}$ as the $r_{k}(k=1,2)$ determined in phase one, and iteratively decrease $l_{1}$ or $l_{2}$ by one, choosing the one that corresponds to the larger probability to the left, $f_{k, l_{k}-1}$, until the leftward error bound is no larger than $\delta_{1}$, where $\delta_{1} \geq 0$ is a specified tolerance. The output is a truncation $\mathbf{n}=\left(l_{1}, r_{1}, l_{2}, r_{2}\right)$ and the numbers $\zeta_{\mathbf{n}}, \eta_{\mathbf{n}}$, and $\theta_{\mathbf{n}}$. For any $\delta_{\mathrm{r}}>0$ and $\delta_{1}>0$, there exist finite $r_{k}$ as required; then $\underline{\sigma}_{1, r_{1}} \underline{\sigma}_{2, r_{2}}>0$, and there exist finite $l_{k}$ as required. The solution to $r_{\mathbf{n}}(\rho)=r^{*}$ (to be computed elsewhere) satisfies (27) and in particular $-\delta_{\mathrm{r}} \leq r(\rho)-r^{*} \leq \delta_{\mathrm{r}}+\delta_{\mathrm{l}}$. By $\delta_{\mathrm{l}}=0$ we will mean no leftward truncation, i.e., $l_{1}=l_{2}=0$.

To see where Algorithm 1 truncates, consider the case $l_{1}=l_{2}=0$ and some $\delta_{\mathrm{r}}>0$. Suppose $r^{*}>0$. In the limit as $\delta_{\mathrm{r}} \rightarrow 0$, the only requirement is $\theta_{\mathbf{n}} \leq \delta_{\mathrm{r}}$, and we have seen that $\theta_{\mathbf{n}} \sim$ $b_{1} t_{1, r_{1}}^{2}+b_{2} t_{2, r_{2}}^{2}$. with $b_{k}$ defined in point (ii) following Proposition 2. It is easy to see that $t_{1, r_{1}} \sim$ $t_{2, r_{2}} \sim \sqrt{\delta_{\mathrm{r}} /\left(b_{1}+b_{2}\right)}$, so $r_{k}$ is simply the quantile of $F_{k}$ corresponding to this tail probability. The case $r^{*}<0$ gives similar behavior.

\section{The Mixed Problem}

The mixed correlation-matching problem refers to solving $r(\rho)=r^{*}$ where $F_{1}$ is discrete and $F_{2}$ is continuous. This indexing involves no loss of generality. The discrete support points are $0,1,2, \ldots$; 


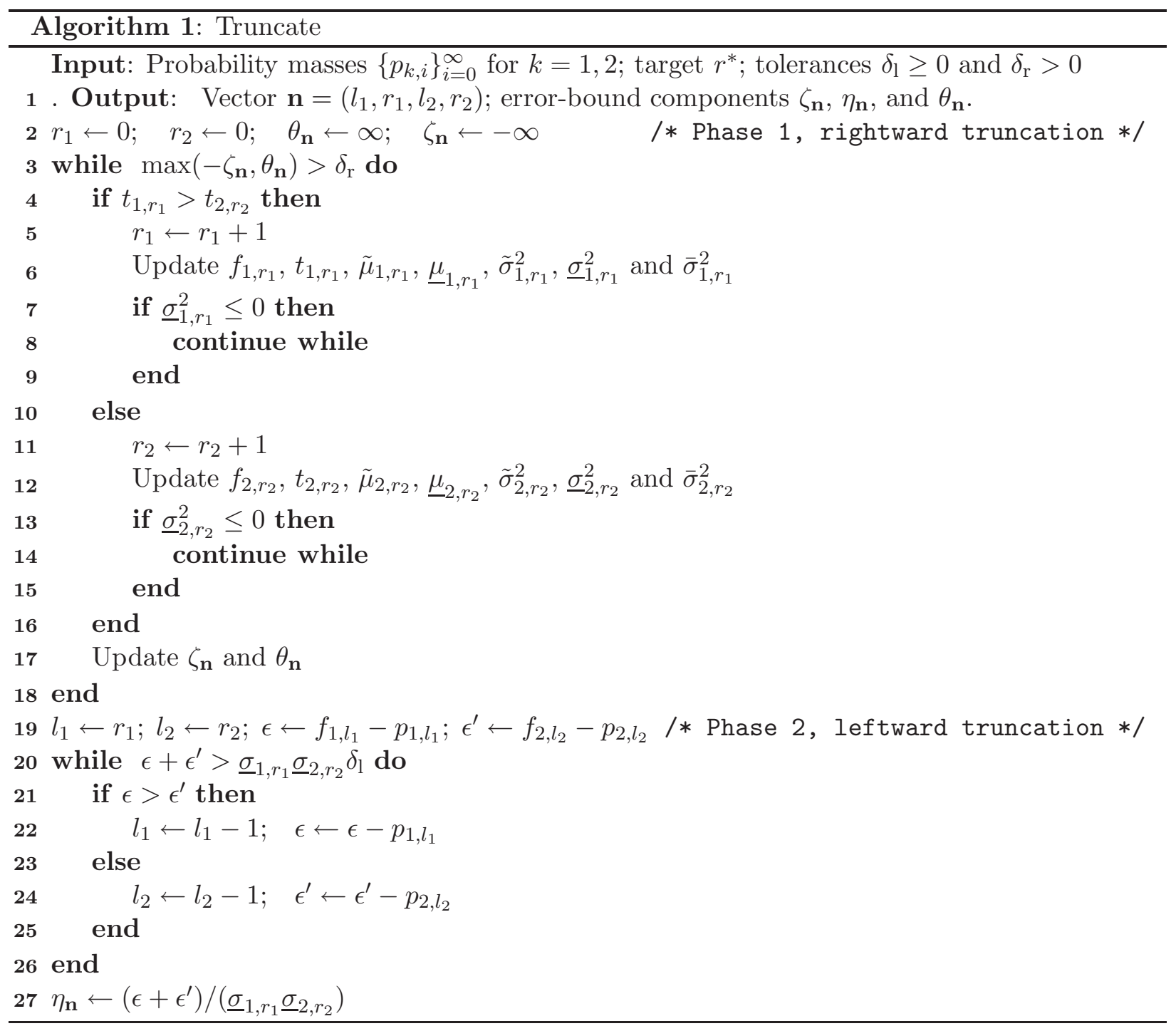


$p_{i}$ is the probability mass at $i$; and $f_{i}=\sum_{j=0}^{i} p_{j}$. The continuity of $F_{2}$ means that $F_{2}\left(X_{2}\right)$ is uniformly distributed on $(0,1)$, so its mean is $\mu_{2}=1 / 2$ and its variance is $\sigma_{2}^{2}=1 / 12$.

The general expression (8) of $g$ would lead to bivariate normal integrals. A more convenient expression is

$$
g(\rho)=\sum_{i=0}^{\infty} f_{i} \int_{0}^{1} u[\Phi(i, u)-\Phi(i-1, u)] \mathrm{d} u,
$$

where $\Phi(i, u)=\Phi(i, u, \rho)=\Phi\left(\left(z_{i}-\rho \Phi^{-1}(u)\right) / \sqrt{1-\rho^{2}}\right)$, with $z_{i}=\Phi^{-1}\left(f_{i}\right)$ and $z_{-1}=-\infty$. This is not difficult to see, and agrees with equation (9) in Channouf and L'Ecuyer (2009), except that the support there is unbounded in both directions.

We will develop an approximation of (30) and associated error bounds. We will then develop an approximation of $r(\rho)$ and error bounds in analogy to the discrete problem. Put $\bar{\Phi}(i, u)=1-\Phi(i, u)$ and $f_{-1}=0$. Rewrite $(30)$ as $g(\rho)=\int_{0}^{1} I(u, \rho) \mathrm{d} u$, where

$$
\begin{aligned}
I(u, \rho) & =u \sum_{i=0}^{\infty} f_{i}[\Phi(i, u)-\Phi(i-1, u)]=u \sum_{i=0}^{\infty} f_{i}[\bar{\Phi}(i-1, u)-\bar{\Phi}(i, u)] \\
& =u \sum_{i=0}^{\infty} \bar{\Phi}(i-1, u)\left(f_{i}-f_{i-1}\right)=u \sum_{i=0}^{\infty} \bar{\Phi}(i-1, u) p_{i} .
\end{aligned}
$$

For an integer $n$, we truncate the sum expression of the integrand $I$ to obtain

$$
I_{n}(u, \rho)=u \sum_{i=0}^{n} \bar{\Phi}(i-1, u, \rho) p_{i}
$$

and we approximate $g(\rho)$ by

$$
\tilde{g}_{n}(\rho)=\int_{0}^{1} I_{n}(u, \rho) \mathrm{d} u
$$

We do not consider truncation to the left for simplicity and because our numerical evidence suggests that the mixed problem is less demanding computationally than the discrete one. To bound the error, observe that

$$
I(u, \rho)-I_{n}(u, \rho)=u \sum_{i>n} \bar{\Phi}(i-1, u, \rho) p_{i} \geq 0 .
$$

Integrating this over $u$, we obtain lower and upper bounds on the error:

$$
0 \leq g(\rho)-\tilde{g}_{n}(\rho)=\int_{0}^{1} u \sum_{i>n} \bar{\Phi}(i-1, u, \rho) p_{i} \mathrm{~d} u \leq t_{n} \int_{0}^{1} u \bar{\Phi}(n, u, \rho) \mathrm{d} u \leq \frac{t_{n}}{2} .
$$

Computing the integral upper bound above (second from the right) would require numerical integration. For simplicity, we will forego this and use instead the looser upper bound on the right.

Let $t_{1, n}, \tilde{\mu}_{1, n}, \underline{\mu}_{1, n}, \tilde{\sigma}_{1, n}, \underline{\sigma}_{1, n}$, and $\bar{\sigma}_{1, n}$ be as in Section 3.2 with truncation point $n$, and referring to the discrete marginal. Put $\tilde{r}_{n}(\rho)=\left(\tilde{g}_{n}(\rho)-\tilde{\mu}_{1, n} / 2\right) /\left(\tilde{\sigma}_{1, n} / \sqrt{12}\right)$ as an approximation of $r(\rho)$. Since $\tilde{g}_{n}$ is the $g$ in (30) that results when we shift to the point $n$ the probability mass 
of the points to its right, it follows immediately from Corollary 1 that $\tilde{r}_{n}$ is a continuous strictly increasing function on $[-1,1]$, and thus has an inverse; that is, for $r^{*} \in\left[\tilde{r}_{n}(-1), \tilde{r}_{n}(1)\right]$, there exists a unique $\rho$ such that $\tilde{r}_{n}(\rho)=r^{*}$, which we denote $\tilde{r}_{n}^{-1}\left(r^{*}\right)$. Our main result is as follows.

Proposition 3 Let $\rho^{*}=\tilde{r}_{n}^{-1}\left(r^{*}\right)$, where $r^{*} \in\left[\tilde{r}_{n}(-1), \tilde{r}_{n}(1)\right]$. Provided that $\underline{\sigma}_{1, n}^{2}$ is positive, we have

$$
\zeta_{n} \leq r\left(\rho^{*}\right)-r^{*} \leq \theta_{n} \quad \text { for all } n
$$

where

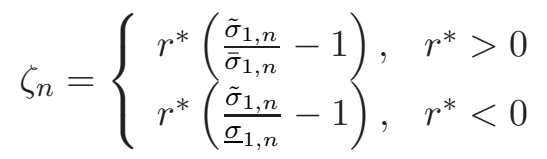

and

$$
\theta_{n}= \begin{cases}\frac{\sqrt{12}\left(t_{1, n}+\tilde{\mu}_{1, n}-\underline{\mu}_{1, n}\right)}{2 \underline{\sigma}_{1, n}}+r^{*}\left(\frac{\tilde{\sigma}_{1, n}}{\underline{\sigma}_{1, n}}-1\right), & r^{*}>0 \\ \frac{\sqrt{12}\left(t_{1, n}+\tilde{\mu}_{1, n}-\underline{\mu}_{1, n}\right)}{2 \underline{\sigma}_{1, n}}+r^{*}\left(\frac{\tilde{\sigma}_{1, n}}{\bar{\sigma}_{1, n}}-1\right), & r^{*}<0 .\end{cases}
$$

Proof. Putting $\tilde{h}_{n}(y)=\tilde{g}_{n}(y)-\tilde{\mu}_{1, n} / 2-r^{*} \tilde{\sigma}_{1, n} / \sqrt{12}$, we have $\tilde{h}_{n}\left(\rho^{*}\right)=0$. Equation (28) holds, where $\tilde{\mu}_{2, .}=\mu_{2}=1 / 2$ and $\tilde{\sigma}_{2, \cdot}=\sigma_{2}=1 / \sqrt{12}$ refer to the continuous marginal. The result now follows from (34), (14), and (15).

Note that $\zeta_{n} \leq 0$ and $\theta_{n}>0$. We can see the asymptotics of the error bounds in (35) as $n \rightarrow \infty$, which will show that the error converges to zero. The quantity $\zeta_{n}$ behaves according to point (i) following Proposition 2, modified to eliminate the tail corresponding to the continuous marginal, so $\zeta_{n}=O\left(t_{1, n}^{2}\right)$. A simple calculation gives $\theta_{n}=\left[\sqrt{12} /\left(2 \sigma^{2}\right)\right] t_{1, n}+o\left(t_{1, n}\right)$. The asymptotic differs from that in the discrete case because the bounding method here is different.

Remark 2 The bounds in (34), (14), and (15) imply lower and upper bounds on $r(\rho)$ analogous to (29). The distance between these bounds converges to zero as $n \rightarrow \infty$. This enables the computation of $r(\rho)$, for any $\rho \in[-1,1]$, to any desired accuracy.

The work to solve $\tilde{r}_{n}(\rho)=r^{*}$ tends to be roughly linear in $n$ as a consequence of the work to compute $I_{n}(u, \rho)$ being (roughly) linear. Our approach parallels that for the discrete problem: initialize $n$ as the smallest support point and iteratively increase it by one until $\max \left(-\zeta_{n}, \theta_{n}\right)$ is at most a specified tolerance $\delta$; for any $\delta>0$, clearly there exists a finite $n$ satisfying this.

\section{$5 \quad$ Numerical Results}

We solved test problems with marginals in one of three families: discrete Pareto, Poisson, and negative binomial. For these problems, solutions to equations $\tilde{r}_{\mathbf{n}}(\rho)=r^{*}$ associated to two different truncations, that is, different $\mathbf{n}$, are computed, as detailed later. The root may be computed via standard derivative-free methods (we use MATLAB's fzero) or via derivative-based ones as in 
Avramidis et al. (2009). On average, the derivative-based methods were slightly faster. For the mixed problem, we report on the derivative-free method, simply to avoid having to give (integral) expressions for the derivatives. Computations were done in MATLAB, and CPU times measured via tic/toc commands. We do not claim these times are competitive; for example, in solving a few problems from Avramidis et al. (2009) with identical truncation and root-finder, our CPU times are larger by a factor of about one thousand. The large timing gap seems to be primarily due to the computer language (these authors use Java). We compute $\bar{\Phi}_{\rho}(x, y)$, the standard bivariate-normal c.d.f. at $(-x,-y)$, via MATLAB's function mvncdf to tolerance $10^{-9}$; this method cites Drezner and Wesolowsky (1989), so we think it is reasonably efficient.

Discrete and mixed problems appear in Sections 5.1 and 5.2, respectively.

\subsection{Discrete Problems}

For the Pareto and Poisson families, four values $r^{*}$ are chosen between $0.999 \tilde{r}_{\mathbf{n}_{\mathbf{0}}}(-0.9999)$ and $0.999 \tilde{r}_{\mathbf{n}_{0}}(0.9999)$, that is, close to the minimal and maximal rank correlation, respectively, inclusively of these and in equal distance. The benchmark uses the truncation vector $\mathbf{n}_{\mathbf{0}}=\left(l_{1,0}, r_{1,0}, l_{2,0}, r_{2,0}\right)$, where $l_{k, 0}$ is the leftmost support point and $r_{k, 0}$ is the quantile of order $1-p$, where $p=10^{-6}$. This $p$ value is also the choice of Avramidis et al. (2009) and Channouf and L'Ecuyer (2009). We compare this against truncation via Algorithm 1 with tolerances specified shortly. The root-finding problem is solved by a hybrid of the Newton-Raphson method and bisection, identical to Press et al. (1992, routine rtsafe, pp. 366-367) and to method NI3 in Avramidis et al. (2009, Section 3.1.4), to which we refer for analytical derivatives of $g_{\mathbf{n}}$.

A user of Algorithm 1 with (absolute error) tolerance $\delta$ will choose a number $0<\beta \leq 1$ and set $\delta_{\mathrm{r}}=\delta \beta$ and $\delta_{\mathrm{l}}=\delta(1-\beta)$. We considered $\beta \in\{1 / 10,1 / 2,1\}$ to assess potential sensitivity. In the Pareto case, with $\delta=10^{-3}, \beta=1$ was optimum (minimized both number of terms and CPU time). This case is shown in Table 1 in detail, but efficiency (the ratio of the benchmark's CPU time to our CPU time) is high for all $\beta$ (the average efficiency of the best $\beta$ to the worst one is roughly 3). Each of the six panels in Table 1 specifies a pair of marginals and the benchmark number $w_{0}=\left(r_{1,0}-l_{1,0}+1\right)\left(r_{2,0}-l_{2,0}+1\right)$. Each row within a panel corresponds to the problem instance with target $r^{*}$; we report the (approximate) solution $\rho$; our method's number $w=\left(r_{1}-l_{1}+1\right)\left(r_{2}-l_{2}+1\right)$; our CPU time; the error estimate $\tilde{r}_{\mathbf{n}_{\mathbf{0}}}(\rho)-r^{*}$ (where "3e-04" means $3 \times 10^{-4}$ ); and the efficiency. Heavier tails (smaller $\alpha$ ) are associated with more work for our method (larger $w$ and CPU) and larger efficiency. Efficiency is also reflected well by the ratio $w_{0} / w$. The work reduction is a result of adhering to the accuracy requirement via the bounds, together with the accuracy being modest. With increased accuracy, we can expect work to increase; for example, for $\delta_{\mathrm{r}}=10^{-6}$ and $\delta_{\mathrm{l}}=0$, average efficiency in the six panels drops to 1.9, 2.8, 5.2, 4.3, 9.4, and 23.5, respectively. Incidentally, in the first row of each panel, the target is $r^{*}=0.999 r_{\mathbf{n}_{0}}(-0.9999)$ and 
the (approximate) solution $\rho$ is far from -0.9999 ; this happens because the function $r_{\mathbf{n}_{\mathbf{0}}}$ increases very slowly between -0.9999 and that $\rho$. We also solved the same problems with a second root finder, MATLAB's fzero; efficiencies were (again) roughly linear in $w_{0} / w$.

Table 1: Discrete problem with $\operatorname{Pareto}\left(\alpha_{1}\right)$ and Pareto $\left(\alpha_{2}\right)$ marginals. $\delta_{\mathrm{r}}=10^{-3}, \delta_{1}=0$.

\begin{tabular}{lrrrrrr}
\hline & $r^{*}$ & $\rho$ & $w$ & $\mathrm{CPU}(\mathrm{sec})$ & $\tilde{r}_{\mathbf{n}_{\mathbf{0}}}(\rho)-r^{*}$ & efficiency \\
\hline$\alpha_{1}=5, \alpha_{2}=5$ & -0.0368 & -0.5160 & 49 & 0.20 & $3 \mathrm{e}-04$ & 16.5 \\
$w_{0}=484$ & 0.3044 & 0.6541 & 49 & 0.19 & $3 \mathrm{e}-04$ & 10.9 \\
& 0.6455 & 0.9157 & 49 & 0.32 & $3 \mathrm{e}-04$ & 11.2 \\
& 0.9867 & 0.9999 & 49 & 0.56 & $3 \mathrm{e}-04$ & 11.2 \\
\hline$\alpha_{1}=5, \alpha_{2}=4$ & -0.0547 & -0.5677 & 72 & 0.33 & $4 \mathrm{e}-04$ & 29.9 \\
$w_{0}=1496$ & 0.2001 & 0.4849 & 72 & 0.24 & $4 \mathrm{e}-04$ & 23.4 \\
& 0.4550 & 0.7892 & 72 & 0.32 & $5 \mathrm{e}-04$ & 24.8 \\
& 0.7099 & 0.9923 & 72 & 0.51 & $5 \mathrm{e}-04$ & 22.1 \\
\hline$\alpha_{1}=5, \alpha_{2}=3$ & -0.0846 & -0.6420 & 190 & 0.95 & $5 \mathrm{e}-04$ & 112.6 \\
$w_{0}=14190$ & 0.1311 & 0.3341 & 190 & 0.74 & $5 \mathrm{e}-04$ & 85.5 \\
& 0.3468 & 0.6875 & 190 & 0.74 & $5 \mathrm{e}-04$ & 85.7 \\
& 0.5625 & 0.9999 & 190 & 1.18 & $6 \mathrm{e}-04$ & 80.7 \\
\hline$\alpha_{1}=4, \alpha_{2}=4$ & -0.0815 & -0.6277 & 100 & 0.53 & $5 \mathrm{e}-04$ & 65.4 \\
$w_{0}=4624$ & 0.2752 & 0.5436 & 100 & 0.39 & $5 \mathrm{e}-04$ & 53.6 \\
& 0.6319 & 0.8777 & 100 & 0.60 & $5 \mathrm{e}-04$ & 52.8 \\
& 0.9887 & 0.9999 & 100 & 1.18 & $5 \mathrm{e}-04$ & 53.5 \\
\hline$\alpha_{1}=4, \alpha_{2}=3$ & -0.1259 & -0.7134 & 261 & 1.63 & $4 \mathrm{e}-04$ & 205.1 \\
$w_{0}=43860$ & 0.1659 & 0.3426 & 261 & 1.11 & $4 \mathrm{e}-04$ & 183.5 \\
& 0.4576 & 0.7269 & 261 & 1.10 & $5 \mathrm{e}-04$ & 183.7 \\
& 0.7494 & 0.9987 & 261 & 1.97 & $5 \mathrm{e}-04$ & 170.6 \\
\hline$\alpha_{1}=3, \alpha_{2}=3$ & -0.1945 & -0.7882 & 529 & 3.36 & $5 \mathrm{e}-04$ & 952.8 \\
$w_{0}=416025$ & 0.2008 & 0.3475 & 529 & 2.35 & $5 \mathrm{e}-04$ & 828.7 \\
& 0.5960 & 0.7933 & 552 & 2.82 & $5 \mathrm{e}-04$ & 803.8 \\
& 0.9913 & 0.9999 & 552 & 7.15 & $5 \mathrm{e}-04$ & 809.8 \\
\hline
\end{tabular}

The second set of examples has Poisson marginals. We keep $\delta=10^{-3}$, and show in Table 2 the (preferred) case $\beta=1 / 2$. For $\beta=1 / 10$, efficiency is between 1.9 and 5.1, and averages 4.9 in the last panel. For $\beta=1$, efficiency is between 1.4 and 6.5 , except for the last row, where it is 0.83 despite the fact that $w<w_{0}$.

For the negative-binomial marginals and targets in Avramidis et al. (2009), performance is comparable to the Poisson case. In the largest problems (largest means), $w_{0}$ is about 190 thousand, $w$ is 28,27 , and 57 thousand for $\beta=1 / 10,1 / 2$, and 1 , respectively, and efficiency is about $w_{0} / w$.

The work of Algorithm 1 was not significant as a fraction of the overall work. In Table 1, this fraction averaged $0.7 \%$, and the maximum was $4.5 \%$; in Table 2 , the respective figures were $1.3 \%$ and $9.6 \%$. The larger fractions occurred consistently in the problems requiring less work. 
Table 2: Discrete problem with Poisson $\left(\lambda_{1}\right)$ and Poisson $\left(\lambda_{2}\right)$ marginals. $\delta_{\mathrm{r}}=\delta_{1}=0.5 \times 10^{-3}$.

\begin{tabular}{lrrrrrr}
\hline & $r^{*}$ & $\rho$ & $w$ & CPU $(\mathrm{sec})$ & $\tilde{r}_{\mathbf{n}_{\mathbf{0}}}(\rho)-r^{*}$ & efficiency \\
\hline$\lambda_{1}=1$ & -0.8501 & -0.9898 & 30 & 0.18 & $9 \mathrm{e}-05$ & 3.8 \\
$\lambda_{2}=1$ & -0.2359 & -0.2922 & 30 & 0.11 & $1 \mathrm{e}-04$ & 3.5 \\
$w_{0}=100$ & 0.3783 & 0.4635 & 30 & 0.09 & $2 \mathrm{e}-04$ & 3.5 \\
& 0.9925 & 0.9999 & 30 & 0.39 & $3 \mathrm{e}-04$ & 3.0 \\
\hline$\lambda_{1}=1$ & -0.9248 & -0.9963 & 100 & 0.69 & $7 \mathrm{e}-05$ & 3.2 \\
$\lambda_{2}=10$ & -0.3075 & -0.3505 & 100 & 0.32 & $1 \mathrm{e}-04$ & 3.1 \\
$w_{0}=290$ & 0.3099 & 0.3539 & 100 & 0.32 & $3 \mathrm{e}-04$ & 3.1 \\
& 0.9272 & 0.9981 & 100 & 0.63 & $4 \mathrm{e}-04$ & 3.3 \\
\hline$\lambda_{1}=1$ & -0.9352 & -0.9987 & 330 & 2.07 & $3 \mathrm{e}-04$ & 5.6 \\
$\lambda_{2}=100$ & -0.3116 & -0.3532 & 330 & 1.08 & $4 \mathrm{e}-04$ & 5.2 \\
$w_{0}=1520$ & 0.3121 & 0.3550 & 330 & 1.05 & $6 \mathrm{e}-04$ & 5.0 \\
& 0.9358 & 0.9997 & 330 & 2.69 & $6 \mathrm{e}-04$ & 4.3 \\
\hline$\lambda_{1}=10$ & -0.9818 & -0.9985 & 400 & 2.85 & $1 \mathrm{e}-05$ & 2.1 \\
$\lambda_{2}=10$ & -0.3222 & -0.3394 & 400 & 1.44 & $9 \mathrm{e}-05$ & 2.2 \\
$w_{0}=841$ & 0.3374 & 0.3549 & 400 & 1.42 & $2 \mathrm{e}-04$ & 2.1 \\
& 0.9970 & 0.9998 & 400 & 4.71 & $3 \mathrm{e}-04$ & 2.2 \\
\hline$\lambda_{1}=10$ & -0.9906 & -0.9987 & 1300 & 9.65 & $2 \mathrm{e}-04$ & 3.3 \\
$\lambda_{2}=100$ & -0.3294 & -0.3450 & 1300 & 4.93 & $3 \mathrm{e}-04$ & 3.4 \\
$w_{0}=4408$ & 0.3317 & 0.3478 & 1300 & 4.89 & $5 \mathrm{e}-04$ & 3.4 \\
& 0.9928 & 0.9993 & 1320 & 10.82 & $5 \mathrm{e}-04$ & 3.0 \\
\hline$\lambda_{1}=100$ & -0.9972 & -0.9988 & 4422 & 34.70 & $2 \mathrm{e}-04$ & 5.1 \\
$\lambda_{2}=100$ & -0.3320 & -0.3460 & 4422 & 17.47 & $3 \mathrm{e}-04$ & 5.1 \\
$w_{0}=23104$ & 0.3332 & 0.3479 & 4422 & 17.23 & $4 \mathrm{e}-04$ & 5.1 \\
& 0.9984 & 0.9996 & 4489 & 41.71 & $4 \mathrm{e}-04$ & 5.1 \\
\hline
\end{tabular}

\subsection{Mixed Problems}

We compare two alternative truncation points: (i) a benchmark $n_{0}$, set as the quantile of order $1-10^{-6}$; and (ii) the smallest $n$ such that the error bound $\max \left(-\zeta_{n}, \theta_{n}\right)$ is no larger than $\delta=10^{-3}$. The values $r^{*}$ are chosen via near-extremes $0.999 \tilde{r}_{n_{0}}( \pm 0.9999)$, as before. The respective equations, $\tilde{r}_{n_{0}}(\rho)=r^{*}$ and $r_{n}(\rho)=r^{*}$, are solved with MATLAB's fzero, described as "a combination of bisection, secant, and inverse quadratic interpolation methods". The integral in (33) is evaluated via MATLAB's quadgk function, described as "adaptive quadrature based on a Gauss-Kronrod pair (15th- and 7th-order formulas)", with error tolerance $10^{-12}$.

Mixed problems whose discrete marginal is Pareto are seen in Table 3. Heavier tails are associated with more work for our method (larger $n$ and CPU), and larger efficiency, which is also reflected well by $n_{0} / n$. The mixed problem is less demanding than the discrete one with same marginals, in agreement with Channouf and L'Ecuyer (2009). 
Table 3: Mixed problem with a $\operatorname{Pareto}(\alpha)$ discrete marginal. $\delta=10^{-3}$.

\begin{tabular}{lrrrrrr}
\hline & $r^{*}$ & $\rho$ & $n$ & CPU $(\mathrm{sec})$ & $\tilde{r}_{n_{0}}(\rho)-r^{*}$ & efficiency \\
\hline$\alpha=5$ & -0.3204 & -0.9970 & 16 & 0.02 & $-4 \mathrm{e}-10$ & 1.3 \\
$n_{0}=22$ & -0.1068 & -0.2606 & 16 & 0.02 & $-3 \mathrm{e}-10$ & 1.2 \\
& 0.1068 & 0.2606 & 16 & 0.03 & $6 \mathrm{e}-09$ & 1.1 \\
& 0.3205 & 0.9971 & 16 & 0.03 & $-1 \mathrm{e}-08$ & 1.2 \\
\hline$\alpha=4$ & -0.4580 & -0.9981 & 32 & 0.03 & $-1 \mathrm{e}-09$ & 1.5 \\
$n_{0}=68$ & -0.1527 & -0.2884 & 32 & 0.03 & $-3 \mathrm{e}-07$ & 1.6 \\
& 0.1527 & 0.2884 & 32 & 0.04 & $3 \mathrm{e}-07$ & 1.6 \\
& 0.4580 & 0.9981 & 32 & 0.05 & $3 \mathrm{e}-08$ & 1.6 \\
\hline$\alpha=3$ & -0.6465 & -0.9987 & 126 & 0.11 & $-3 \mathrm{e}-08$ & 4.5 \\
$n_{0}=645$ & -0.2155 & -0.3194 & 126 & 0.11 & $2 \mathrm{e}-08$ & 4.8 \\
& 0.2155 & 0.3194 & 126 & 0.18 & $3 \mathrm{e}-08$ & 5.1 \\
& 0.6465 & 0.9987 & 126 & 0.18 & $-1 \mathrm{e}-07$ & 5.0 \\
\hline$\alpha=2.2$ & -0.8254 & -0.9990 & 2055 & 2.34 & $1 \mathrm{e}-07$ & 32.7 \\
$n_{0}=61597$ & -0.2751 & -0.3416 & 2055 & 2.39 & $3 \mathrm{e}-08$ & 32.7 \\
& 0.2751 & 0.3416 & 2055 & 4.76 & $9 \mathrm{e}-08$ & 33.2 \\
& 0.8254 & 0.9990 & 2055 & 4.70 & $1 \mathrm{e}-07$ & 33.9 \\
\hline
\end{tabular}

\section{Conclusion}

We contributed to the mathematics of constructing a random vector $\mathbf{X}$ of the form (1) by controlling, separately for each pair of coordinates of $\mathbf{X}$, the rank correlations or product-moment correlations. For arbitrary univariate distribution functions $F_{1}$ and $F_{2}$, we gave expressions for $\mathbb{E}\left[F_{1}\left(X_{1}\right) F_{2}\left(X_{2}\right)\right]$ and $\mathbb{E}\left[X_{1} X_{2}\right]$ and their derivatives with respect to $\rho$ and showed that both the rank correlation $r(\rho)$ and the product-moment correlation are differentiable strictly increasing functions on $(-1,1)$, thus proving existence and uniqueness of the solution for any feasible target. For the case where $r(\rho)$ cannot be computed exactly due to an infinite discrete support, we showed how to construct an approximation $\tilde{r}$ of $r$ such that equations of form $r(\rho)=r^{*}$ can be solved to any desired accuracy.

In addition to ensuring accuracy, our method may require less work than truncating at quantiles $x_{p}$ associated to a small tail probability $p$, because the work decreases as the (absolute error) tolerance increases. With the tolerance fixed, higher efficiency seems to result by setting $\beta$ (Section 5.1) depending on the probability mass functions (p.m.f.'s): if both p.m.f.'s are nonincreasing in the direction of the infinite right tail (example: Pareto), truncate only the tail: $\beta=1$; if both p.m.f.'s are (approximately) symmetric, for example normal-like, due to a central-limit effect (examples: large-mean Poisson; negative binomial with large "number of failures" parameter), set $\beta=1 / 2$. In other cases, $\beta=1 / 2$ seems reasonable, though not necessarily most efficient. Heavier tails, that is, higher sensitivity of $x_{p}$ to $p$, seem to translate to higher potential for work reduction.

Some ideas for future inquiry are now proposed. Marginals with large mean(s) tend to result 
in large $w$ and according work, even with our approach. More efficient solution of such problems is an open research problem. Another line of inquiry could be to see if our approach can be extended to the product-moment correlation for general discrete and unbounded marginals. A difficulty in this program is that the summands in the corresponding infinite sums do not seem to permit the convenient bound " 1 " that we used for the cumulative probabilities.

\section{Acknowledgments}

The author thanks Professor Pierre L'Ecuyer for his comments on an early draft of the paper.

\section{References}

Asmussen, S., P.W. Glynn. 2007. Stochastic Simulation: Algorithms and Analysis. Springer, New York.

Avramidis, A. N. 2009. Fitting discrete multivariate distributions with unbounded marginals and normal-copula dependence. M. D. Rossetti, R. R. Hill, B. Johansson, A. Dunkin, R. G. Ingalls, eds., Proceedings of the 2009 Winter Simulation Conference. Institute of Electrical and Electronics Engineers, Inc., Piscataway, New Jersey, 452-458.

Avramidis, A. N., N. Channouf, P. L'Ecuyer. 2009. Efficient correlation matching for fitting discrete multivariate distributions with arbitrary marginals and normal-copula dependence. INFORMS Journal on Computing 21 88-106.

Axtell, R. L. 2001. Zipf distribution of U.S. firm sizes. Science 293 1818-1820.

Biller, B., B.L. Nelson. 2003. Modeling and generating multivariate time-series input processes using a vector autoregressive technique. ACM Transactions on Modeling and Computer Simulation 13 211-237.

Cario, M. C., B. L. Nelson. 1996. Autoregressive to anything: Time series input processes for simulation. Operations Research Letters 19 51-58.

Cario, M. C., B. L. Nelson. 1997. Modeling and generating random vectors with arbitrary marginal distributions and correlation matrix. Technical Report, Department of Industrial Engineering and Management Science, Northwestern University.

Channouf, N., P. L'Ecuyer. 2009. Fitting a normal copula for a multivariate distribution with both discrete and continuous marginals. Proceedings of the 2009 Winter Simulation Conference. IEEE Press, 352-358. 
Channouf, N., P. L'Ecuyer. 2012. A normal copula model for the arrival process in a call center. International Transactions in Operational Research 19 771-787.

Deuchert, E., S. Brody. 2007. Plausible and implausible parameters for mathematical modeling of nominal heterosexual HIV transmission. Annals of Epidemiology 17 237-244.

Drezner, Z., G. O. Wesolowsky. 1989. On the computation of the bivariate normal integral. Journal of Statistical Computation and Simulation 35 101-107.

Embrechts, P., A. McNeil, D. Straumann. 2002. Correlation and dependence in risk management: properties and pitfalls. M. A. H. Dempster, ed., Risk Management: Value at Risk and Beyond. Cambridge University Press, Cambridge, 176-223.

Ghosh, S., S.G. Henderson. 2003. Behavior of the NORTA method for correlated random vector generation as the dimension increases. ACM Transactions on Modeling and Computer Simulation $13276-294$.

Joe, H. 2005. Asymptotic efficiency of the two-stage estimation method for copula-based models. Journal of Multivariate Analysis 94401 - 419.

Kruskal, W. 1958. Ordinal measures of association. Journal of the Americal Statistical Association $53814-861$.

Lehmann, E. L. 1966. Some concepts of dependence. Annals of Mathematical Statistics $371137-$ 1153.

Parulekar, Minothi, Armand M. Makowski. 1997. Tail probabilities for M/G/ $\infty$ input processes (I): Preliminary asymptotics. Queueing Systems 27 271-296.

Press, W. H., S. A. Teukolsky, W. T. Vetterling, B. P. Flannery. 1992. Numerical Recipes in C: The Art of Scientific Computing. 2nd ed. Combridge University Press, New York.

Rudin, W. 1976. Principles of Mathematical Analysis. 3rd ed. McGraw-Hill, New York.

Suárez-González, A., J. C. López-Ardao, C. López-García, M. Fernández-Veiga, R. F. R. Rubio, M. E. S. Vieira. 2002. A new heavy-tailed discrete distribution for LRD M/G/ $\infty$ sample generation. Performance Evaluation 47 197-219. 